PROCEEDINGS OF THE

AMERICAN MATHEMATICAL SOCIETY

Volume 136, Number 12, December 2008, Pages 4349-4354

S 0002-9939(08)09409-4

Article electronically published on June 25, 2008

\title{
INCOMPRESSIBILITY OF TORI TRANSVERSE TO AXIOM A FLOWS
}

\author{
C. A. MORALES \\ (Communicated by Jane M. Hawkins)
}

\begin{abstract}
We prove that a torus transverse to an Axiom A vector field that does not exhibit sinks, sources or null homotopic periodic orbits on a closed irreducible 3-manifold is incompressible. This strengthens the works of Brunella (1993), Fenley (1995), and Mosher (1992).
\end{abstract}

\section{INTRODUCTION}

It is well known that an Anosov vector field on a closed atoroidal 3-manifold is transitive. This follows from the fact that a torus transverse to an Anosov vector field on a closed 3-manifold is incompressible [2], 3], 15]. In this paper we extend both results to the more general class of vector fields on irreducible 3-manifolds, namely, Axiom A vector fields that do not exhibit sinks, sources or null homotopic periodic orbits. Recall that a sink of a $C^{1}$ vector field is a hyperbolic attracting closed orbit, while a source is a sink for the time-reversed vector field. See [14] where sufficient conditions for the transitivity of Axiom A vector fields on toroidal 3 -manifolds are given. Let us present our results in a precise way.

Hereafter $M, X$ and $X_{t}$ will denote a 3-manifold, a $C^{1}$ vector field on $M$ and the flow generated by $X$ in $M$ respectively. We say that $M$ is closed if it is compact connected and has empty boundary. We say that $M$ is irreducible if every embedded 2 -sphere in $M$ is the boundary of a 3 -ball in $M$. A two-side surface $S$ in $M$ is incompressible if the homomorphism $\pi_{1}(S) \rightarrow \pi_{1}(M)$ induced by the inclusion is injective. We say that $X$ is transitive if it has a dense orbit. A compact invariant set $H$ of $X$ is hyperbolic if there is a tangent bundle splitting $T_{H} M=E_{H}^{s} \oplus E_{H}^{X} \oplus E_{H}^{u}$ over $H$ such that $E_{H}^{s}$ is contracting, $E_{H}^{u}$ is expanding and $E_{H}^{X}$ is the subbundle generated by $X$. A nonwandering point of $X$ is a point $p \in M$ such that for every neighborhood $U$ of $p$ and every $T>0$ there is $t>T$ such that $X_{t}(U) \cap U \neq \emptyset$. We say that $X$ is Axiom $A$ if its nonwandering set is hyperbolic with dense closed orbits, and Anosov if $M$ is a hyperbolic set. An Anosov vector field is Axiom A but not conversely. See [8, [6, [10, [17] for details. Our main result is the following.

Received by the editors May 22, 2007, and, in revised form, October 3, 2007, and October 24, 2007.

2000 Mathematics Subject Classification. Primary 37D20; Secondary 57M27.

Key words and phrases. Sink, vector field, atoroidal, incompressible torus.

This work was supported in part by CNPq, FAPERJ and PRONEX-Brazil. The author thanks Professors E. Apaza, D. Carrasco-Olivera and B. San Martin for helpful conversations. He also thanks the Instituto de Matemáticas Puras e Aplicadas (IMPA) for its kind hospitality.

(C)2008 American Mathematical Society 
Theorem 1.1. Let $X$ be an Axiom A vector field that does not exhibit sinks, sources or null homotopic periodic orbits on a closed irreducible 3-manifold. Then, every torus transverse to $X$ is incompressible.

Let us state two short corollaries of Theorem 1.1. Recall that a closed 3-manifold is atoroidal if it has no incompressible tori [8, [10].

Corollary 1.1. Let $X$ be an Axiom A vector field that does not exhibit sinks, sources or null homotopic periodic orbits on a closed irreducible 3-manifold $M$. If $M$ is atoroidal, then $X$ is a transitive Anosov vector field.

The following corollary was proved early in [2, [3, [15].

Corollary 1.2. Anosov vector fields on closed atoroidal 3-manifolds are transitive.

Theorem 1.1 becomes false if we remove the hypothesis that $X$ does not exhibit null homotopic periodic orbits [1. On the other hand, if all closed 3-manifolds exhibiting an Axiom A vector field as in the theorem were irreducible, then we could remove the hypothesis that $M$ is irreducible from the theorem. However, nonirreducible closed 3-manifolds exhibiting such Axiom A vector fields do exist [13. Finally, Corollary 1.1 becomes false if we remove the hypothesis that $M$ is atoroidal. Indeed, a counterexample can be constructed as in [4].

\section{Proofs}

A solid torus is a compact 3-manifold diffeomorphic to the product of a 2-disk and a circle. The boundary and the interior of a manifold $U$ will be denoted by $\partial U$ and $\operatorname{Int}(U)$ respectively. The following elementary lemma deals with solid tori inside a solid torus.

Lemma 2.1. Let $S T^{1}$ and $S T^{2}$ be two solid tori contained in a solid torus $S T$. If $\partial\left(S T^{1}\right) \subset \operatorname{Int}\left(S T^{2}\right)$, then $S T^{1} \subset \operatorname{Int}\left(S T^{2}\right)$.

Proof. By contradiction assume that $S T^{1} \not \subset \operatorname{Int}\left(S T^{2}\right)$. We claim that $\partial\left(S T^{2}\right) \subset$ $\operatorname{Int}\left(S T^{1}\right)$. Indeed, $S T^{1} \cap \partial\left(S T^{2}\right) \neq \emptyset$ since $S T^{1}$ is connected and $\partial\left(S T^{2}\right)$ separates $S T$. Note that $S T^{1} \cap \partial\left(S T^{2}\right)$ is closed in $\partial\left(S T^{2}\right)$ since $S T^{1}$ and $\partial\left(S T^{2}\right)$ are closed in $\partial\left(S T^{2}\right)$. Moreover, $S T^{1} \cap \partial\left(S T^{2}\right)$ is open in $\partial\left(S T^{2}\right)$ since $S T^{1} \cap \partial\left(S T^{2}\right)=$ $\operatorname{Int}\left(S T^{1}\right) \cap \partial\left(S T^{2}\right)$. As $\partial\left(S T^{2}\right)$ is connected we conclude that $S T^{1} \cap \partial\left(S T^{2}\right)=$ $\partial\left(S T^{2}\right)$ and then $\partial\left(S T^{2}\right) \subset S T^{1}$. Actually we have $\partial\left(S T^{2}\right) \subset \operatorname{Int}\left(S T^{1}\right)$ since $\partial\left(S T^{1}\right) \subset \operatorname{Int}\left(S T^{2}\right)$, and so $\partial\left(S T^{2}\right) \cap \partial\left(S T^{1}\right)=\emptyset$. This proves the claim.

As $\partial\left(S T^{2}\right) \subset \operatorname{Int}\left(S T^{1}\right)$ (by the claim) and $\partial\left(S T^{1}\right) \subset \operatorname{Int}\left(S T^{2}\right)$ (by hypothesis), we conclude that $S T^{1} \cup S T^{2}$ is a closed 3-manifold. Obviously $S T^{1} \cup S T^{2} \subset S T$, and so $S T^{1} \cup S T^{2}$ is open and closed in $S T$. Since $S T$ is connected we conclude that $S T^{1} \cup S T^{2}=S T$. But this is a contradiction since $S T$ has boundary and $S T^{1} \cup S T^{2}$ does not. The result follows.

The following lemma deals with compact 3-manifolds with toral boundary inside a solid torus.

Lemma 2.2. Let $S T$ be a solid torus and let $U \subset S T$ be a compact connected 3-manifold whose boundary $\partial U=T_{1} \cup \cdots \cup T_{n}$ is a union of tori. If each $T_{i}$ bounds a solid torus $S T_{i}$ in $S T$, then there is $i_{0} \in\{1, \cdots, n\}$ such that $S T_{i} \cap U=T_{i}$, for 
all $i \neq i_{0}$ in $\{1, \cdots, n\}$, and

$$
U \cup\left(\bigcup_{i \in\{1, \cdots, n\}, i \neq i_{0}} S T_{i}\right) \subseteq S T_{i_{0}} .
$$

In particular, if $n=1$, then $U$ is a solid torus.

Proof. Note that each $T_{i}$ divides $S T$ in two connected components, one of which is the solid torus $S T_{i}$. Note also that $\operatorname{Int}(U)$ is contained in one of these components since it is connected and $T_{i} \subset \partial U$. If $\operatorname{Int}(U)$ is not contained in $S T_{i}$, for all $i$, then we would obtain a closed 3-manifold inside $S T$ by just capping each $T_{i}$ with $S T_{i}$. But no such manifolds inside $S T$ exist by the argument in the last part of the proof of Lemma 2.1. So, $U \subset S T_{i_{0}}$ for some $i_{0}$; thus $T_{i} \subset \operatorname{Int}\left(S T_{i_{0}}\right)$ for each $i \neq i_{0}$ in $\{1, \cdots, n\}$. Then, $S T_{i} \subset \operatorname{Int}\left(S T_{i_{0}}\right)$ for all $i \neq i_{0}$ in $\{1, \cdots, n\}$ by Lemma 2.1. This proves the first part of the lemma.

Now assume that $\partial U$ is formed by a single torus $T_{1}$ which bounds a solid torus $S T_{1}$ in $S T$. By the first part we get $i_{0}=1$, hence $U \subset S T_{1}$. Since $S T_{1}$ and $U$ have common boundary $T_{1}$ we conclude that $U$ is an open subset of $S T_{1}$ relative to $S T_{1}$. But $U$ is also a closed subset of $S T_{1}$ relative to $S T_{1}$; therefore $U=S T_{1}$ since $S T_{1}$ is connected. Therefore $U$ is a solid torus since $S T_{1}$ is also. This proves the lemma.

Next we introduce some basic concepts in hyperbolic dynamics [6], 17]. Let $X$ be a $C^{1}$ vector field on a closed 3-manifold $M$. The omega-limit set of $x \in M$ is defined by

$$
\omega(x)=\left\{y=\lim _{n \rightarrow \infty} X_{t_{n}}(x): t_{n} \text { is a sequence converging to } \infty \text { as } n \rightarrow \infty\right\} .
$$

A compact invariant set $\Lambda$ is transitive if $\Lambda=\omega(x)$ for some $x \in \Lambda$. An attractor is a transitive set $\Lambda$ such that

$$
\Lambda=\bigcap_{t \geq 0} X_{t}(U)
$$

for some compact neighborhood $U$. This neighborhood is a basin of attraction of $\Lambda$ if it is a compact 3-manifold with nonempty boundary $\partial U$ transverse to $X$. A proper attractor always has a basin of attraction. A repeller of $X$ is an attractor for its time reversed field $-X$. A basin of repulsion of a repeller $R$ of $X$ is a basin of attraction of $R$ viewed as an attractor of $-X$.

A hyperbolic attractor (resp. hyperbolic repeller) of $X$ is a hyperbolic set which is also an attractor (resp. a repeller) of $X$. In particular, a sink (resp. a source) is a hyperbolic attractor (resp. repeller), but not conversely. It follows from Smale's Spectral Decomposition Theorem that every Axiom A vector field exhibits a hyperbolic attractor and a hyperbolic repeller which are proper if the vector field is not transitive.

On the other hand, if $x \in M$ belongs to a hyperbolic set of $X$, then the so-called Invariant Manifold Theory [9] asserts that the strong stable manifold of $x$ defined by

$$
W^{s s}(x)=\left\{y \in M: \lim _{t \rightarrow \infty} d\left(X_{t}(y), X_{t}(x)\right)=0\right\}
$$

is a $C^{1}$ immersed submanifold of $M$. Consequently, the stable manifold

$$
W^{s}(x)=\bigcup_{t \in \mathbb{R}} W^{s s}\left(X_{t}(x)\right)
$$




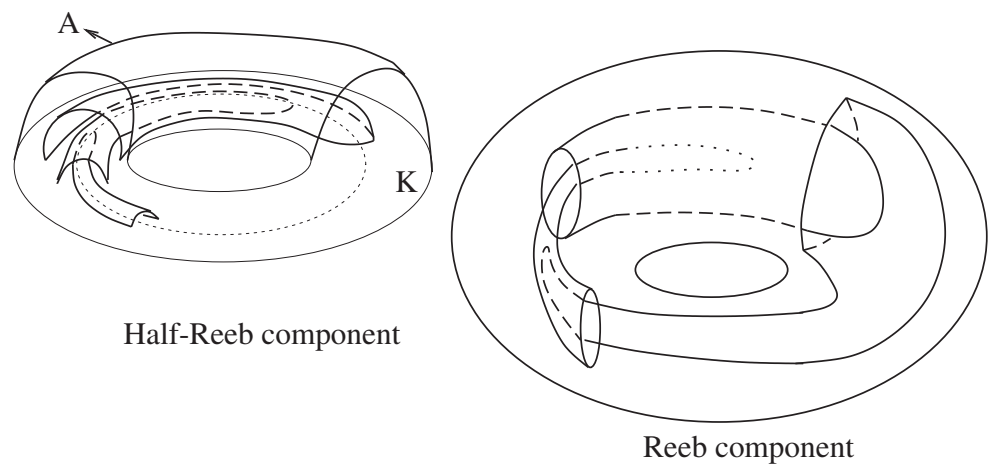

FiguRE 1

is an immersed submanifold as well.

Now we state the concept of a half-Reeb component, which will be useful in the next lemma (for basic concepts in foliation theory including the definition of Reeb component; see [5] and the references therein). Let $\mathcal{F}$ be a foliation in a solid torus $S T$ transverse to $\partial(S T)$. A half-Reeb component of $\mathcal{F}$ is a saturated subset $H \subset S T$, bounded by an annulus leaf $A$ and an annulus $K \subset \partial(S T)$ with $\partial K=\partial A$, such that the double manifold $2 H$ is a Reeb component of the double foliation $2 \mathcal{F}$ (see 2] and Figure 1).

The following lemma was proved in [1]. We outline its proof here for the sake of completeness.

Lemma 2.3. A hyperbolic attractor having a solid torus as a basin of attraction is an attracting periodic orbit (hence it is a sink).

Proof. It suffices to prove that the expanding subbundle of the attractor is zero dimensional. By contradiction we assume that such a subbundle is not zero dimensional. Then, both the stable and unstable subbundles of the attractor are one dimensional. It follows that the stable manifolds $W^{s}(x)$ form an invariant foliation $\mathcal{F}$ in the solid torus which is transverse to the boundary.

Clearly $\mathcal{F}$ has no closed leaves; hence $\mathcal{F}$ has no Reeb components. On the other hand, $\mathcal{F}$ has no half-Reeb components too, due to an argument based on 2. It follows that $\mathcal{F}$ has neither Reeb nor half-Reeb components, and so the double foliation $2 \mathcal{F}$ defined in $S^{2} \times S^{1}$ (the double of the solid torus) has no Reeb components.

But $2 \mathcal{F}$ is supported in $S^{2} \times S^{1}$, which is a manifold with nonzero second homotopy group. So, $2 \mathcal{F}$ is the trivial product foliation of $S^{2} \times S^{1}$ by spheres (e.g. Theorem 1.10-(iii), p. 92 in [5]), and so $\mathcal{F}$ is the trivial product foliation by meridian disks on $S T$. This implies that the leaves of $\mathcal{F}$ are invariant disks. Applying the classical Poincare-Bendixon Theorem [16 to one of these disks, we could find a singularity in the solid torus. However, such singularities cannot exist due to the continuity of the hyperbolic splitting. This contradiction proves the result.

The previous lemmas will be used to prove the following one.

Lemma 2.4. Let $X$ be an Axiom $A$ vector field on a closed 3-manifold $M$. If there is a solid torus in $M$ whose boundary is transverse to $X$, then $X$ exhibits either a sink or a source or a null homotopic periodic orbit. 
Proof. Let $S T$ be the solid torus in the statement of the lemma. We can assume that $X$ points inward to $S T$ in $\partial(S T)$, for, otherwise, we replace $X$ by $-X$ in the argument below. Then, the Spectral Decomposition Theorem [6] implies that there is a hyperbolic attractor $\Lambda$ of $X$ in $S T$. Of course we can assume that $\Lambda$ is not a sink, for, otherwise, we are done. Note that $X$ is not transitive since it points inward to $S T$ in $\partial(S T)$. Therefore $\Lambda$ is proper, and so it exhibits a basin of attraction $U$ contained in $S T$. As $\Lambda$ is not a sink we have that $\partial U$ is a union of tori transverse to $X$.

We claim that if $X$ does not exhibit null homotopic periodic orbits, then every torus in $\partial U$ bounds a solid torus in $S T$. By contradiction, assume that there is a torus in $\partial U$ which does not bound a solid torus in $S T$. As $S T$ is irreducible atoroidal we have that such a torus is contained in a 3-ball in $S T$ (e.g. 8], 10] or (4), p. 11 in [7]). Then such a torus divides $S T$ in two connected components, one of which contained in the ball (see for instance [13]). Hence we could find a periodic orbit inside the ball, a contradiction because $X$ does not exhibit null homotopic periodic orbits. This contradiction proves the claim.

Hereafter we assume that $X$ does not exhibit null homotopic periodic orbits. Then, the previous claim implies that every torus in $\partial U$ bounds a solid torus in $S T$. Therefore we can apply Lemma 2.2 to $U$. Replacing $S T$ by the solid torus $S T_{i_{0}}$ in that lemma, we can assume that $\partial(S T)$ is one of the boundary components of $U$. If $U$ has no more boundary components apart from $\partial(S T)$, then $U$ is a solid torus by Lemma 2.2 since $n=1$ in such a case. So, Lemma 2.3 would imply that $\Lambda$ is a sink, a contradiction since we have assumed that $\Lambda$ is not a sink. Therefore, there is another torus $T^{1}$ in $\partial U$. Set $S T^{0}=S T$ and let $S T^{1}$ be the solid torus bounded by $T^{1}$ in $S T^{0}$ (it exists by the claim). Note that $X$ points outward from $S T^{1}$ at $T^{1}$; therefore there is a repeller $\Lambda^{1}$ in the spectral decomposition of $X$ inside $S T^{1}$. Of course we can assume that $\Lambda^{1}$ is not a source, for, otherwise, we are done.

By repeating the above argument we get a nested sequence of solid tori

$$
S T^{0} \subset S T^{1} \subset S T^{2} \subset \cdots \subset S T^{k} \subset \cdots
$$

with $S T^{i} \backslash \operatorname{Int}\left(S T^{i+1}\right)$ containing a hyperbolic attractor of $X$ (for $i$ even) or a hyperbolic repeller of $X$ (for $i$ odd). As the number of attractors and repellers in the spectral sequence of $X$ is finite, we have that the sequence must stop. But this occurs precisely when some of the solid tori $S T^{k}$ is the basin of attraction (or repulsion) of a hyperbolic attractor (or repeller) of $X$. Therefore, $X$ exhibits a sink or a source by Lemma 2.3. This finishes the proof.

Proof of Theorem 1.1, Let $T$ be a torus transverse to an Axiom A vector field $X$ in a closed irreducible 3-manifold. In addition, suppose that $X$ does not exhibit sinks, sources or null homotopic periodic orbits. As the manifold is irreducible we have that $T$ either bounds a solid torus or is contained in a 3-ball or is incompressible. If $T$ bounds a solid torus, then $X$ would exhibit either a sink or a source or a null homotopic periodic orbit by Lemma 2.4. If $T$ is contained in a 3-ball, then there is a periodic orbit in the ball, and so $X$ would exhibit a null homotopic periodic orbit. In both cases we get a contradiction; therefore $T$ is incompressible. This finishes the proof.

Proof of Corollary 1.1, Let $X$ be an Axiom A vector field that does not exhibit sinks, sources or null homotopic periodic orbits on a closed irreducible atoroidal 3manifold. Let us prove that $X$ is transitive. By contradiction assume that it is not 
so. Then, $X$ exhibits a proper hyperbolic attractor in its spectral decomposition. Because such an attractor is proper we have that it has a basin of attraction $U$. But the attractor cannot be a sink by hypothesis, so $\partial U$ is formed by finitely many disjoint tori transverse to $X$. Then, there is an incompressible torus by Theorem 1.1, a contradiction since the manifold is atoroidal. This contradiction proves that $X$ is transitive. As every transitive Axiom A vector field is Anosov we get the result.

Proof of Corollary 1.2. The result follows directly from Corollary 1.1, since every Anosov vector field on a closed atoroidal 3-manifold satisfies the hypotheses of Corollary 1.1.

\section{REFERENCES}

[1] Apaza., E., Soares, R., Axiom A flows without sinks nor sources on 3-manifolds, Discrete Contin. Dyn. Syst. 21 (2008), no. 2, 393-401.

[2] Brunella, M., Separating the basic sets of a nontransitive Anosov flow, Bull. London Math. Soc. 25 (1993), no. 5, 487-490. MR.1233413 (94g:58163)

[3] Fenley, S. R., Quasigeodesic Anosov flows and homotopic properties of flow lines, J. Differential Geom. 41 (1995), no. 2, 479-514. MR1331975 (96f:58118)

[4] Franks, J., Williams, B., Anomalous Anosov flows, Global theory of dynamical systems (Proc. Internat. Conf., Northwestern Univ., Evanston, Ill., 1979), pp. 158-174, Lecture Notes in Math., 819, Springer, Berlin, 1980. MR591182 (82e:58078)

[5] Gabai, D., 3 lectures on foliations and laminations on 3-manifolds, Laminations and foliations in dynamics, geometry and topology (Stony Brook, NY, 1998), 87-109, Contemp. Math., 269, Amer. Math. Soc., Providence, RI, 2001. MR1810537(2002g:57032)

[6] Hasselblatt, B., Katok, A., Introduction to the modern theory of dynamical systems. With a supplementary chapter by Katok and Leonardo Mendoza, Encyclopedia of Mathematics and Its Applications, 54, Cambridge University Press, Cambridge, 1995. MR1326374|(96c:58055)

[7] Hatcher, A., Notes on Basic 3-Manifold Topology, available at Hatcher's homepage.

[8] Hempel, J., 3-Manifolds, Ann. of Math. Studies, No. 86, Princeton University Press, Princeton, N.J.; University of Tokyo Press, Tokyo, 1976. MR0415619 (54:3702)

[9] Hirsch, M., Pugh, C., Shub, M., Invariant Manifolds, Lec. Not. in Math. 583, Springer-Verlag, 1977. MR0501173 (58:18595)

[10] Jaco, W., Lectures on Three-Manifold Topology, CBMS Regional Conference Series in Mathematics, 43, American Mathematical Society, Providence, R.I., 1980. MR.565450 (81k:57009)

[11] Morales, C., Poincaré-Hopf index and partial hyperbolicity, Ann. Fac. Sci. Toulouse Math. XVII (2008), no. 1, 193-206.

[12] Morales, C., Examples of singular-hyperbolic attracting sets, Dyn. Syst. 22 (2007), no. 3, 339-349.

[13] Morales, C., Incompressibility of torus transverse to vector fields, Spring Topology and Dynamical Systems Conference. Topology Proc. 28 (2004), no. 1, 219-228. MR2105459 (2005j:57028)

[14] Morales, C., Axiom A flows with a transverse torus, Trans. Amer. Math. Soc. 355 (2003), no. 2, 735-745. MR:1932723 (2003g:37049)

[15] Mosher, L., Dynamical systems and the homology norm of a 3-manifold. I. Efficient intersection of surfaces and flows, Duke Math. J. 65 (1992), no. 3, 449-500. MR.1154179(93g:57018a)

[16] Palis, J., de Melo, W., Geometric theory of dynamical systems. An introduction. Translated from the Portuguese by A. K. Manning. Springer-Verlag, New York- Berlin, 1982. MR669541 (84a:58004)

[17] Robinson, C., Dynamical Systems. Stability, Symbolic Dynamics, and Chaos, Second edition. Studies in Advanced Mathematics, CRC Press, Boca Raton, FL, 1999. MR 1792240 (2001k:37003)

Instituto de Matemática, Universidade Federal do Rio de Janeiro, P. O. Box 68530, 21945-970 Rio DE JANEIRO, BRAZIL

E-mail address: morales@impa.br 